\title{
El Bosquejo geográfico de la provincia de Zaragoza, la base cartográfica para el Plan General de Caminos Vecinales de 1900
}

\author{
José Luis Villanova \\ Universitat de Girona. Departament de Geografia \\ josel.villanova@udg.edu
}

\section{Resumen}

Este artículo analiza, a partir de documentación original y en parte inédita, las fuentes y el proceso de formación del Bosquejo geográfico de la provincia de Zaragoza, que sería la base cartográfica para diseñar el Plan General de Caminos Vecinales que la Diputación Provincial de Zaragoza aprobó en 1900. Su autor fue el diputado provincial Dionisio Casañal, reputado topógrafo que había realizado excelentes trabajos topográficos y cartográficos. Con muy escasos medios económicos, pero con un gran conocimiento de la provincia y de los materiales cartográficos preexistentes, Casañal consiguió elaborar, con mínimos trabajos de campo, un documento que fue aceptado unánimemente por la Diputación. Además, su autor también fue el impulsor del citado plan de caminos vecinales, cuyo objetivo era sacar del aislamiento a numerosos pueblos que tenían serias dificultades para poder comercializar adecuadamente sus productos agrícolas.

Palabras clave: historia de la cartografía; provincia de Zaragoza; Dionisio Casañal; caminos vecinales.

* El presente artículo ha sido realizado en el marco del proyecto de investigación ref. CSO2014-54078-C2-1-P, financiado por el Ministerio de Economía y Competitividad. Aprovecho la ocasión para agradecer a Luis Urteaga sus valiosos comentarios durante la redacción del mismo, y a Luis Magallanes, Francisco J. Dávila y Álvaro Lafuente, las facilidades para la digitalización de cartografía en los archivos del Centro Geográfico del Ejército, el Instituto Geográfico Nacional y la Diputación Provincial de Zaragoza, respectivamente. 
Resum. El Bosquejo geográfico de la provincia de Zaragoza, la base cartogràfica per al Pla General de Camins Veïnals de 1900

L'article analitza, a partir de documentació original i en part inèdita, les fonts i el procés de formació del Bosquejo geográfico de la provincia de Zaragoza, que seria la base cartogràfica per dissenyar el Pla General de Camins Veïnals que la Diputació Provincial de Saragossa va aprovar l'any 1900. L'autor en va ser el diputat provincial Dionisio Casañal, reputat topògraf que havia realitzat excel-lents treballs topogràfics i cartogràfics. Amb uns mitjans econòmics insignificants, però amb un gran coneixement de la província i dels materials cartogràfics preexistents, Casañal va aconseguir elaborar, amb ben pocs treballs de camp, un document que va ser acceptat unànimement per la Diputació. A més, el seu autor també fou l'impulsor de l'esmentat pla de camins veïnals, l'objectiu del qual era treure de l'aïllament nombrosos pobles que patien serioses dificultats per poder comercialitzar adequadament els seus productes agrícoles.

Paraules clau: història de la cartografia; província de Saragossa; Dionisio Casañal; camins veïnals.

Résumé. Le Bosquejo geográfico de la provincia de Zaragoza: la base cartographique pour le Plan général de chemins vicinaux de 1900

L'article analyse, à partir d'une documentation originale et en partie inédite, les sources et le processus de formation du Bosquejo geográfico de la provincia de Zaragoza; la base cartographique utilisée pour préparer le Plan général de chemins vicinaux, approuvé par la Diputación Provincial de Zaragoza en 1900. Son auteur a été le député provincial Dionisio Casañal, topographe célèbre qui avait réalisé d'excellents travaux topographiques et cartographiques. Disposant de peu moyens économiques, mais avec une grande connaissance de la province et des matériels préexistants, Casañal a formé un document que la Diputación a accepté à l'unanimité. En outre, son auteur a aussi été le promoteur du ledit plan de chemins vicinaux. Son objectif était de sortir de l'isolement de nombreux villages qui rencontraient de sérieuses difficultés pour pouvoir commercialiser leurs produits agricoles de façon convenable.

Mots-clés: histoire de la cartographie; province de Saragosse; Dionisio Casañal; chemins vicinaux.

Abstract. The Bosquejo geográfico de la provincia de Zaragoza: The cartographic base for the Local Roads Plan of 1900

Using original and in part unpublished documents, this paper discusses the sources and the process to develop the Bosquejo geográfico de la provincia de Zaragoza; the cartographic base for the Local Roads Plan approved by the Provincial Council of Saragossa in 1900. The author of the plan was the provincial legislator Dionisio Casañal, a highly reputed surveyor who had performed excellent survey and cartographic works. With very little economic resources, but a vast knowledge of the province and pre-existing cartographic works, Casañal created a map that was accepted unanimously by the provincial council. In addition, he was also the promoter of the Local Roads Plan to bring out of isolation many villages that faced enormous difficulties to trade their agricultural products.

Keywords: history of cartography; Saragossa province; Dionisio Casañal; local roads. 


\section{Sumario}
1. Introducción
4. Los resultados
2. La propuesta de Dionisio Casañal
5. Conclusiones
3. Las fuentes utilizadas por Casañal
Referencias bibliográficas

\section{Introducción}

A pesar de la compleja relación entre infraestructuras y crecimiento económico, está fuera de toda duda que el crecimiento económico de un país puede verse entorpecido por una mala red de comunicaciones y que el desarrollo de las vías de comunicación puede facilitar el de la vida económica. Ahora bien, la necesaria articulación del mercado interior a finales del siglo xix, en una sociedad eminentemente agraria como la española, dependía tanto de la existencia de una extensa red de comunicaciones nacionales como de la densidad de la malla provincial y local (Fontana, 1986).

A finales del siglo xIx, existía en España una red bastante extensa de carreteras del Estado, más de $30.000 \mathrm{~km}$. Por el contrario, el panorama en la red secundaria era mucho menos alentador. La longitud de las carreteras provinciales no llegaba a $7.000 \mathrm{~km}$ y la de los caminos vecinales ${ }^{1}$ rondaba los $3.400 \mathrm{~km}$, aunque este último dato debe tomarse con cautela, pues la información sobre los mismos es muy deficiente. De todas formas, «la conclusión unánime de todos los estudiosos del momento era: existen en España suficientes carreteras del estado y faltan, en gran manera, carreteras provinciales y aún más caminos vecinales» (Uriol Salcedo, 1992: 69). La escasez de vías secundarias se debía, fundamentalmente, a que su construcción y conservación eran responsabilidad de las diputaciones y los ayuntamientos, instituciones que no contaban con los medios ni con los recursos económicos suficientes para llevar a cabo los proyectos y las obras. Además, el Estado debía aprobar los planes y los proyectos y no había considerado prioritarias estas actuaciones (Alzola y Minondo, 1899; Gómez Mendoza, 1991; Font i Garolera, 1999; Herranz Loncan, 2004).

Tal estado de cosas también se reproducía en la provincia de Zaragoza, que presentaba una densidad de la red similar a la media española, tanto en lo que respecta a carreteras del Estado como a carreteras provinciales y caminos

1. El Real Decreto de 7-4-1848 estableció que se entenderían por caminos vecinales «los caminos públicos» que no estuvieran comprendidos en "las clases de carreteras nacionales o provinciales», y los clasificó en caminos de primer orden — «los que por conducir a un mercado, a una carretera nacional o provincial, a un canal, a la capital de un distrito judicial o electoral, o por cualquier otra circunstancia interesen a varios pueblos a un tiempo, y sean de un tránsito activo y frecuente»— y de segundo orden — los que, interesando a uno o más pueblos a la vez, fueran poco transitados «por carecer de un objeto especial» que les diera importancia- (art. 1. ${ }^{\circ}$ ). Aunque la legislación posterior del siglo XIX volvió a referirse a estos caminos de diferentes formas, la decisión de la Diputación Provincial de Zaragoza de recurrir a esta norma legal para definir el Plan General de Caminos Vecinales de 1900 induce a pensar que el concepto seguía vigente en aquellos momentos. 
vecinales (Herranz Loncan, 2008). Esta última deficiencia era especialmente grave en una provincia caracterizada por una economía de base agraria, baja densidad de población y poblamiento muy disperso (Germán Zubero, 1999a y 2012). Además, a mediados de la década de 1890, la economía de la provincia había tocado fondo como consecuencia de la crisis agraria finisecular, en la que había influido muy negativamente "la escasez de carreteras y comunicaciones», como apuntaban los registradores de la propiedad (Germán y Forcadell, 1988: 82; Pinilla Navarro, 1995; Germán Zubero, 2012).

Y fue en este contexto, en 1895 concretamente, cuando Dionisio Casañal presentó su propuesta a la Diputación Provincial de Zaragoza (DPZ) para formar «una carta geográfica de la provincia» que sirviese de base a "un proyecto general de caminos vecinales» para poner remedio al deficiente estado de las comunicaciones en la provincia, circunstancia que dificultaba la comercialización de la producción agraria ${ }^{2}$. La propuesta — que se analizará detalladamente más adelante- obedecía a la necesidad de contar con un mapa provincial para poder realizar un proyecto general de planeamiento viario. Es bien sabido que "las grandes intervenciones territoriales acometidas en el siglo XIX [...] tenían un requisito en común: el empleo de mapas y planos muy precisos», y las tareas de planeamiento viario, en concreto, precisaban de «mapas topográficos a gran escala» (Urteaga y Nadal, 2007: 401). En España, el proyecto de formación del Mapa Topográfico Nacional, a escala 1:50.000, nació a mediados del siglo, y las primeras hojas fueron publicadas en 1875, pero el ritmo de los trabajos fue extremadamente lento. En la década de 1890, solo había aparecido el 10\% de las más de 1.100 hojas previstas. Por lo que respecta a la provincia de Zaragoza, la primera que cubría una parte del territorio (hoja n. ${ }^{\circ}$ 464-Used) no vio la luz hasta 1917 y la publicación de la última (hoja n. ${ }^{\circ}$ 408-Torrijo) tardaría más de 20 años (Urteaga y Nadal, 2001: 259 y 269). Esta circunstancia provocaría que el mapa topográfico no pudiera utilizarse, «ni siquiera como documento de apoyo, en ninguna de las grandes operaciones de ingeniería civil acometidas en España en la segunda mitad del siglo XIX y las primeras décadas del XX», ni tampoco, evidentemente, en la provincia de Zaragoza. Así pues, «la carencia de una carta de base perjudicó las tareas de planificación de la administración pública» $y$, entre otros efectos, "frenó la obtención de mapas corográficos» provinciales, mapas absolutamente necesarios, tanto para las delegaciones del Ministerio de Fomento como para las diputaciones, que tenían competencias sobre las carreteras provinciales y los caminos vecinales. El primer mapa provincial del Instituto Geográfico, el correspondiente a Murcia, no apareció hasta 1905 (Urteaga y Nadal, 2007: 403 y 417) y el de Zaragoza tuvo que esperar hasta $1936^{3}$.

La historia de la cartografía en España cuenta con un considerable número de trabajos sobre los aspectos catastral, urbano y militar, pero son muy pocos

2. Archivo de la Diputación Provincial de Zaragoza (en adelante, ADPZ), Libro de actas, 1895, sign. 61.

3. Ver Instituto Geográfico Nacional, Cartoteca, sign. 13-B-22. 
los referidos a la red viaria. Además, no existe ninguna monografía referida a los mapas provinciales. Entre las excepciones, no todas centradas estrictamente en la historia de la cartografía viaria, pero que, como mínimo, contienen abundantes referencias al respecto, merecen citarse los trabajos sobre Galicia (Nárdiz Ortiz, 1992), el Camino de Santiago (Soria y Puig, 1993), el norte de España (Soria y Puig y Menéndez de Luarca, 1994), el noroeste de la península Ibérica (Menéndez de Luarca y Navía Osorio, 2000), la provincia de Barcelona (Navas y Ferrer, 2007 y 2012) o la evolución de las carreteras españolas (Rodríguez Lázaro et al., 2007) ${ }^{4}$. Sin embargo, carecemos por el momento tanto de una perspectiva general como de una monografía relativa a Aragón.

El presente artículo intenta llenar un pequeño hueco en este ámbito: el análisis de la formación del Bosquejo geográfico de la provincia de Zaragoza, documento que sería la base cartográfica para diseñar un plan general de caminos vecinales. En primer lugar, se analiza la propuesta de Casañal para formar dicha carta geográfica. A continuación, se examinan las fuentes utilizadas para la elaboración del documento. Y, por último, se presentan los resultados finales del trabajo cartográfico de Casañal, acompañándolos de unas breves referencias a los resultados del Plan General de Caminos Vecinales para el que el Bosquejo sirvió de base.

\section{La propuesta de Dionisio Casañal}

Dionisio Casañal y Zapatero (Zaragoza, 1846-1913) fue un topógrafo extraordinario y muy prolífico que, tras trabajar 10 años al servicio del Estado, desarrolló una extensa carrera profesional en la esfera privada. Sus trabajos más conocidos son los planos urbanos de cinco capitales de provincia (Zaragoza, Pamplona, Córdoba, Vitoria y Huesca), pero también levantó numerosos planos parcelarios en el valle del Ebro y deslindó muchos términos municipales. Para desarrollar su actividad profesional, creó el Centro Geodésico-Topográfico de Zaragoza, cuyos trabajos fueron profusamente reconocidos en la época. Junto a su extensa actividad cartográfica, Casañal también tuvo una presencia notable en la vida social y política zaragozana de finales del siglo xIx y principios del xx (Villanova, 2011a, 2011b y 2014; Villanova et al., 2015).

En abril de 1895, Casañal, siendo diputado provincial y vicepresidente de la Comisión de Fomento, se ofreció a la DPZ para formar, en el plazo de cuatro años, «una carta geográfica de la provincia» que sirviera de base cartográfica para diseñar un plan general de caminos vecinales, que también se comprometía a elaborar. Casañal presentó la propuesta por dos razones. Por una parte, consideraba que la falta de un «completo sistema de comunicaciones para el

4. También merece citarse la exposición «Xarxes de comunicació a Catalunya: Passat present i futur», organizada por la Càtedra d'Història de la Comunicació de la Universitat Autònoma de Barcelona, a finales de 1998 e inicios de 1999, en el Institut Cartogràfic de Catalunya. En la misma, se mostraban numerosos mapas que recogían la evolución histórica de las comunicaciones desde la antigüedad, incluidas las viarias, en Cataluña, especialmente, y España. Ver Moreno Sardà (1998). 
transporte de los productos de su suelo» era uno de los problemas de mayor trascendencia para la provincia y que, por lo tanto, había que solucionarlo lo más rápidamente posible. Como la construcción de carreteras provinciales sería extremadamente lenta y gravosa para las arcas de la Diputación, proponía disenar con relativa urgencia un plan general de caminos vecinales que conectasen pueblos incomunicados con vías férreas o carreteras para poder dar salida a su producción agraria. Por otra parte, la ausencia de mapas relativamente recientes de la provincia con las informaciones necesarias para alcanzar dicho objetivo convertía en imprescindible la formación de la carta ${ }^{5}$. Ahora bien, Casañal no contemplaba la posibilidad de formar un mapa topográfico - tarea extremadamente compleja y costosa material y económicamente-, sino simplemente un croquis, un "bosquejo», en el que se consignasen aquellas informaciones imprescindibles para lograr el objetivo propuesto: vías de comunicación, poblados, sus alturas sobre el nivel del mar, cursos de agua, líneas divisorias principales, etc. Dichos datos los obtendría a partir de fuentes cartográficas ya existentes y serían complementados con los que poseían las compañías ferroviarias, las oficinas de Obras Públicas y el Centro Geodésico-Topográfico, además de con los trabajos de campo imprescindibles. Respecto de la retribución económica, solo reclamaba los gastos ocasionados por el movimiento de personal y la traslación de material en las operaciones de campo. Al mes siguiente, la Diputación aprobó la propuesta al considerar muy importante el trabajo para la provincia y el pequeño presupuesto que exigía: el coste calculado solo ascendía a 8.000 pesetas 6 .

La propuesta suponía una auténtica novedad en la provincia, que no contaba con cartografía actualizada que sirviera de base para llevar a cabo operaciones de planificación territorial, como un plan general de caminos vecinales. Pero no lo era tanto a nivel estatal. Por ejemplo, la Diputación Provincial de Barcelona, pionera en este tipo de operaciones, había realizado un mapa de la provincia en 1873 —en el marco de los proyectos de organización y planificación territorial del entonces presidente, Ildefons Cerdà-, que se convertiría en «la base cartográfica imprescindible» para este tipo de trabajos durante años. $\mathrm{Su}$ aplicación más inmediata fue para dibujar las carreteras provinciales del plan presentado por Melcior de Palau en 1878 (Navas y Ferrer, 2007: 111). Es posible que Casañal tuviera conocimiento de este documento gracias a los contactos que había mantenido en Barcelona. Entre 1878 y 1881, había sido codirector, junto a Enrique Berrocal, jefe de los trabajos estadísticos de la provincia de Barcelona, de la Revista Geográfica y Estadística, editada en aquella ciudad (Villanova et al., 2015).

5. A mediados de la década de 1880, la DPZ se había dirigido al Instituto Geográfico y Estadístico (IGE) para gestionar la formación de un mapa topográfico de la provincia. El elevado gasto económico, 580.000 pesetas, había llevado a la institución a desistir de su intención. ADPZ, Libro de actas, 1895, sign. 61.

6. ADPZ, Libro de actas, 1895, sign. 61. 


\section{Las fuentes utilizadas por Casañal}

Como se acaba de apuntar, el documento cartográfico sobre el que pudiera diseñarse el Plan General de Caminos Vecinales no era necesario que reuniese todas las características de un mapa topográfico. Por ello, Casañal decidió utilizar documentos y datos ya existentes para realizarlo, con lo cual limitó al máximo las operaciones de campo. Además, el presupuesto aprobado era muy pequeño y sus ocupaciones profesionales, sociales y políticas, así como las actividades del Centro Geodésico-Topográfico, no debían permitirle dedicar demasiados esfuerzos al proyecto ${ }^{7}$. En este punto, cabe remarcar que el mapa de la provincia de Barcelona de 1873 tampoco se había realizado a partir de operaciones de campo. Había sido ejecutado tomando como base el mapa de la provincia de 1872 que se utilizó para la reforma de los partidos judiciales, cuya base cartográfica, a su vez, pudo ser el mapa de la provincia de 1867 —lamentablemente extraviado-, elaborado por el director de Estudios de Carreteras y Caminos Vecinales, Mariano Oms y Novau, y que, según su autor, puede ser considerada la primera «representación gráfica de la provincia de carácter científico" (Navas y Ferrer, 2007: 105 y 2012). A la vista de la información contenida en el Bosquejo, Casañal utilizó el Atlas de España y sus Posesiones de Ultramar, de Francisco Coello; las Memorias, del IGE; el Mapa Itinerario Militar, y los datos que poseían las compañías ferroviarias, las oficinas de Obras Públicas y el Centro Geodésico-Topográfico, aunque de sus propias palabras se desprende que empleó alguna otra referencia, como se verá más adelante.

\subsection{El Atlas de España y sus posesiones de Ultramar}

La más antigua de las fuentes eran las dos hojas de la provincia de Zaragoza del Atlas, una de las cuales había sido publicada en 1853, la que representaba la pequeña zona de la provincia situada por encima del paralelo $42^{\circ} 12^{\prime}$, y la otra, probablemente, en 1865 (figura 1).

Ambas hojas habían sido elaboradas hacía varias décadas, y las informaciones sobre las comunicaciones terrestres - ferrocarriles construidos y en construcción, caminos reales o arrecifes con hitos o leguarios, caminos carreteros, de herradura y sendas, y puentes, barcas y vados - se encontraban desfasadas. Sin embargo, otras características y otros contenidos lo convirtieron en una obra de referencia fundamental para Casañal. Las hojas incluían la delimitación de los partidos judiciales, a partir de los cuales se diseñaría el Plan General de Caminos Vecinales de la provincia de Zaragoza. La escala, 1:200.000, permitía «una inserción exhaustiva de topónimos referidos a núcleos de población», que se encuentran correctamente localizados. El relieve está representado de forma arbitraria, con curvas de nivel figuradas, y su mayor o menor entidad

7. En la segunda mitad de la década de 1890, el Centro Geodésico-Topográfico levantó planos parcelarios de, al menos, nueve municipios de Zaragoza y Navarra, y publicó una segunda edición del plano de Zaragoza de 1880 con las modificaciones urbanísticas que había experimentado la ciudad (Villanova, 2014). 


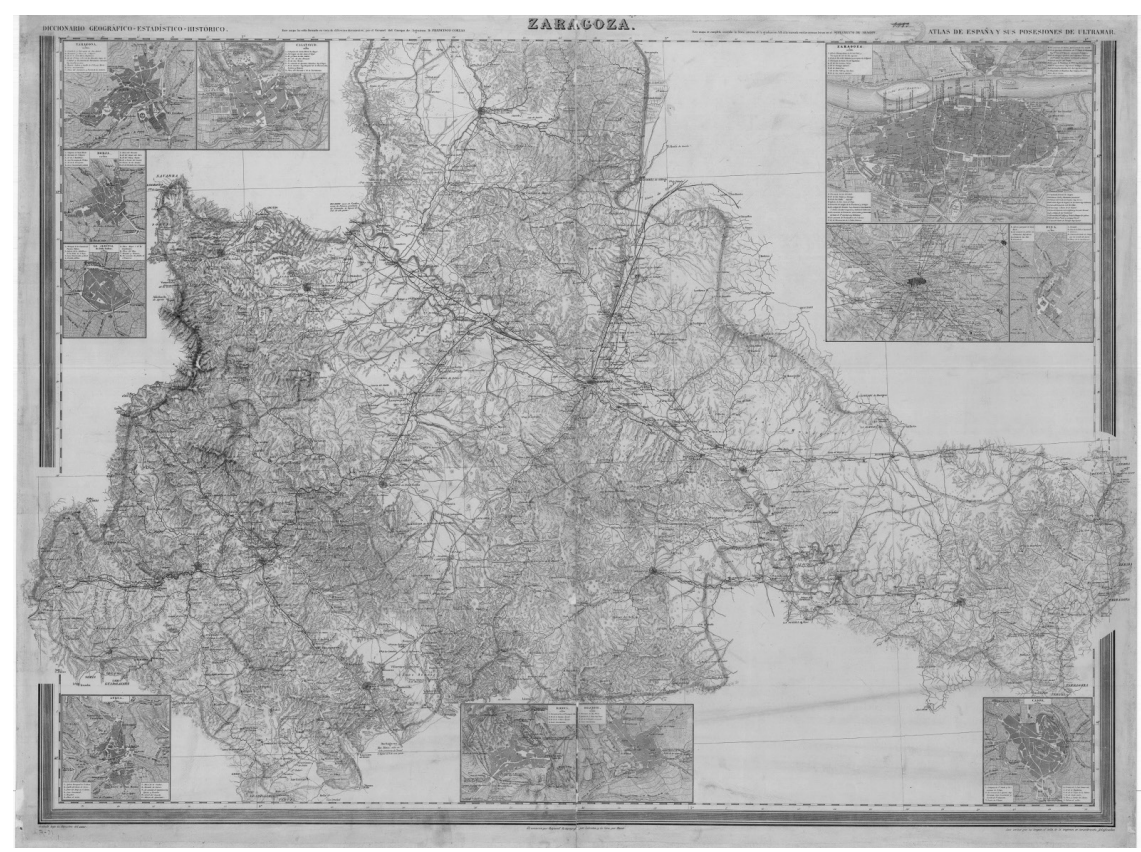

Figura 1. Zaragoza (hoja n. ${ }^{\circ}$ 2) (1865?), 1:200.000, Atlas de España y sus posesiones de Ultramar.

Fuente: Instituto Geográfico Nacional, Cartoteca, sign. 30-C-8.

se diferencia mediante el uso de tres tipos de letra. Esta deficiencia «está en parte compensada por una abundancia de orónimos que hacen de las hojas del Atlas de Coello un hito en la fijación de la toponimia de ese género en la cartografía de España». Por último, la red fluvial, representada minuciosamente y jerarquizada, permitió definir numerosas divisorias de aguas y, «mediante el uso de curvas de nivel figuradas (es decir, aparentes o fingidas), mejorar la representación del relieve, aunque con referencias altimétricas solamente ocasionales» (Gómez Pérez, 1971; Quirós Linares, 2010: 69 y 72).

\subsection{El Mapa itinerario militar de España}

La segunda de las fuentes fueron las hojas números in y viI del Mapa itinerario militar de España, a escala 1:500.000 (figura 2).

Este mapa había sido publicado por el Depósito de la Guerra en 1865, aprovechando los itinerarios gráficos descriptivos para la marcha de las tropas que el Cuerpo de E. M. del Ejército había comenzado a levantar en 1847, y que habían sido completados y revisados en 1863. Incluía, entre otras informaciones que no interesan al objeto de este estudio, las pobla- 


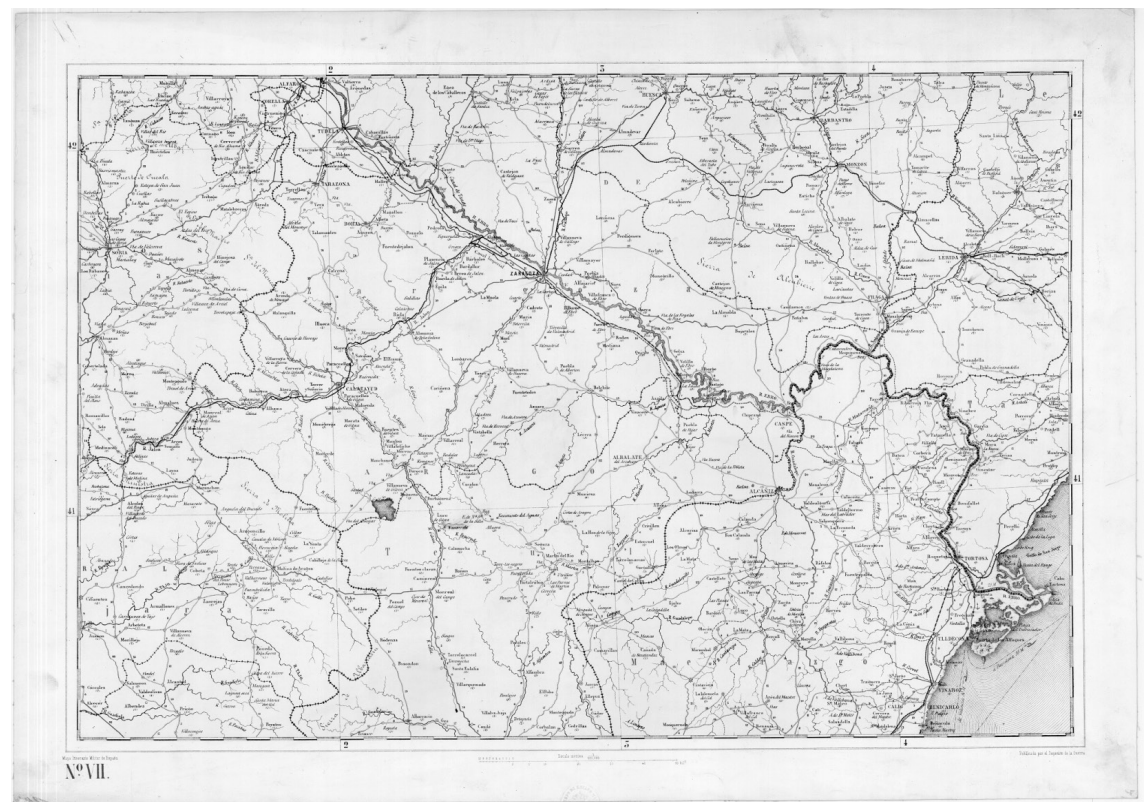

Figura 2. Hoja número VII del Mapa itinerario militar de España (1865), 1:500.000.

Fuente: Archivo Cartográfico del Centro Geográfico del Ejército, sign. 97 Bis VII.

ciones de tránsito, las inmediatas que pudieran tener relación con él y las de cierta importancia — diferenciando su jerarquía—, así como edificios aislados notables que se encontraran en el tránsito; los caminos que las unían - carreteras construidas y en construcción, caminos carreteros, de herradura, sendas y caminos no reconocidos- y las distancias a que se encontraban; la red ferroviaria - aunque solo se indicaba el trazado general de las líneas, extraído de los planos de los proyectos y de las obras de las empresas concesionarias, pues no habían sido reconocidas y muchas se encontraban en construcción-; la hidrografía — convenientemente clasificada, distinguiendo cursos constantes o accidentales-, así como los pasos y los tipos (puentes, barcas y vados), y los nombres de las cordilleras y los puntos más señalados de las líneas divisorias que conviniera distinguir (García-Baquero, 1981; Magallanes Pernas, 2014).

El documento presentaba importantes insuficiencias para los objetivos del Bosquejo. La escala había obligado a suprimir datos de interés, como algunos pasos de ríos, para no hacerlo excesivamente confuso. La información sobre carreteras y caminos procedía de reconocimientos efectuados entre 1847 y 1864 , y solo habían vuelto a reconocerse los más importantes que habían sufrido variaciones notables en su trazado. No se recogía información sobre todas estas vías, sino solamente de aquellas que presentaban 
interés para la marcha de las tropas. Tampoco se situaban todas las poblaciones, como ya se ha señalado, ni tenía altimetría. Y los detalles de los ríos habían sido tomados de cartas publicadas sin excesiva garantía (GarcíaBaquero, 1981).

Pero es posible que Casañal completara estas informaciones con las del Mapa militar itinerario de España, a escala 1:200.000. En octubre de 1898, presentó los trabajos realizados hasta la fecha: los correspondientes a todos los partidos judiciales con excepción de los de Ejea y Sos, y la zona septentrional de los de Tarazona y Borja. Y manifestó que cartografiaría estas regiones tan pronto como el Cuerpo de E. M. hubiera ultimado los trabajos «del mapa militar en la región Norte de esta provincia» ${ }^{8}$. Su comentario induce a pensar que se refería a este mapa, que había comenzado a formarse en 1882 con objeto de completar el anterior con mayor exactitud y garantía. En esta ocasión, por ejemplo, se recogieron todos los caminos susceptibles de ser utilizados en las operaciones de guerra, fueron reconocidos todos los caminos entre pueblos o núcleos de población de cierta importancia y se representaron los ferrocarriles construidos y todas las poblaciones. Tampoco tenía altimetría, pero incluía los accidentes notables del terreno y los cursos de agua a partir de estudios anteriores o de otros datos de contrastada exactitud. Las distancias se especificaban del mismo modo que en el Mapa itinerario militar (García-Baquero, 1981). Al contener más información y más actualizada, es posible que Casañal consultara las hojas correspondientes a la provincia publicadas hasta la finalización del Bosquejo: las de los números 36 y 37 (figura 3), que habían aparecido en 1893 y 1894 , respectivamente, y que abarcaban el sur de la provincia hasta el paralelo $41^{\circ} 36^{\prime}$, pero no pudo utilizar las otras. Las correspondientes a la zona septentrional de la provincia serían publicadas en la década de 1910, y la que recoge una pequeña zona del extremo oriental de la provincia en 1907 (García-Baquero, 1981).

\subsection{Las Memorias del Instituto Geográfico y Estadístico}

La tercera de las fuentes fueron las Memorias del Instituto Geográfico y Estadistico. La serie comenzó a publicarse en 1875, con una periodicidad irregular, y hasta 1899, año en que Casañal terminó su trabajo, habían aparecido un total de 11 volúmenes. Las Memorias contenían los resultados de las numerosas tareas del Instituto, pero, sobre la provincia de Zaragoza, estos volúmenes solo contenían los mapas de los trabajos de establecimiento de la Red Geodésica de Primer Orden y los resultados de las nivelaciones de precisión. De los primeros, Casañal apenas debió tomar la localización de los vértices en la provincia. La información referida a las nivelaciones de precisión le sería de mayor interés, pues se representaban mapas a escala 1:500.000 de las mismas y los itinerarios de las líneas - que se trazaban siguiendo vías férreas y carreteras principales-, sus longitudes y las señales colocadas.

8. ADPZ, Libro de actas, 1898, sign. 64. 


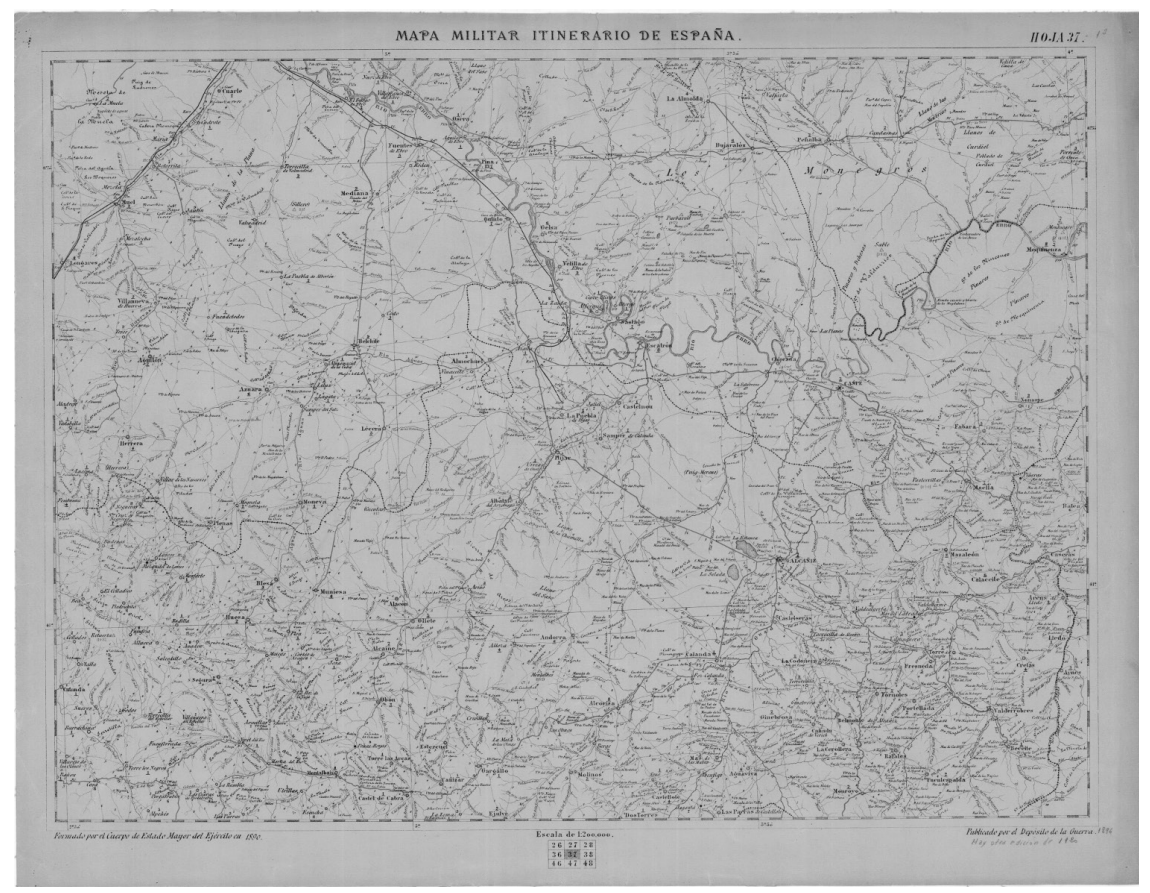

Figura 3. Hoja número 37 del Mapa militar itinerario de España (1894), 1:200.000.

Fuente: Archivo Cartográfico del Centro Geográfico del Ejército, Mapa militar itinerario de España (1894), 1:200.000, hoja número 37.

\subsection{El resto de las fuentes}

Aunque las fuentes descritas, especialmente las dos primeras, proporcionaron a Casañal abundante información, esta no era suficiente para formar el Bosquejo. Se trataba de documentos realizados hacía varias décadas y la información referida a las comunicaciones no era completa. Como la idea directora del Plan General de Caminos Vecinales era conectar los pueblos incomunicados con vías férreas o carreteras, Casañal tuvo que recabar datos actualizados de las compañías ferroviarias y de las oficinas de Obras Públicas.

Desde la década de 1860, Zaragoza se encontraba conectada, mediante líneas de vía ancha, con Barcelona por Lleida, Pamplona y Alsasua por Castejón, y Madrid. Se trataba de líneas que discurrían especialmente por el valle del Ebro, punto de conexión entre las dos regiones más desarrolladas del país, el área de Barcelona y el País Vasco marítimo, y pasaje obligado entre Cataluña y Madrid a través del valle del Jalón. Posteriormente, y hasta finales del siglo, se inauguraron las líneas Tudela-Tarazona, Ariza-Valladolid y Zaragoza-Reus y, en 1901, Calatayud-Sagunto por Teruel (Germán Zubero, 1999c). Paralelamente, se habían construido las líneas de vía estrecha Zaragoza-Cariñena y 
Borja-Cortes, cuya función principal era el transporte de productos agrícolas y mineros (Macías Muñoz, 2005). También existían líneas en construcción o en proyecto (Calatayud-Soria-Santander, Gallur-Sádaba, etc.), aunque algunas (Cariñena-Daroca o Cariñena-Ricla) no llegarían a construirse. Casañal debió consultar los rigurosos mapas del trazado y los perfiles longitudinales de las líneas en los archivos de las compañías que las gestionaban, o las proyectaban, en aquellos momentos: Ferrocarriles del Norte de España, Ferrocarriles de Madrid a Zaragoza y Alicante (MZA), Compañía del Ferrocarril Central de Aragón, Compañía del Ferrocarril de Cariñena a Zaragoza, Sociedad Altos Hornos y Fábrica de Hierro y Acero de Bilbao, Sociedad Anónima Minas y Ferrocarril de Utrillas, etc. (Wais, 1956; Tedde de Lorca, 1978; Germán Zubero, 1999c; Macías Muñoz, 2005)9.

Casañal también recabó informaciones en «las oficinas de Obras Públicas», que tenían en su poder no solo cartografía de los trazados de las carreteras y de los caminos construidos, sino también de los que se encontraban en construcción y en proyecto. Respecto de la denominación tan genérica de las oficinas, puede pensarse que se refería tanto a la Jefatura de Obras Públicas de la provincia, como a la Dirección de Carreteras Provinciales de la DPZ. Desde el Real Decreto de 17-10-1863, que ampliaba las competencias de las entidades provinciales en materia de obras públicas, las diputaciones habían comenzado a realizar planes de carreteras provinciales, creando para ello direcciones que se ocupaban de gestionarlas. Por otra parte, la Ley de carreteras de 4-5-1877 había establecido que las jefaturas provinciales de Obras Públicas fueran las responsables de la elaboración de los proyectos, la construcción y la conservación de las carreteras a cargo del Estado. Las que no estaban incluidas entre éstas corrían a cargo de las provincias, y los caminos vecinales, de los municipios, aunque las jefaturas intervenían en la aprobación de los planes y proyectos elaborados por las diputaciones y los municipios, y ejercían la inspección sobre las obras ejecutadas por ambos entes locales.

La última de las fuentes citada por Casañal fue la de los trabajos del Centro Geográfico-Topográfico; fundamentalmente, los referentes a cartografía parcelaria rústica. Esta es una de las actividades de Casañal menos estudiadas, y solamente existen referencias aisladas, y muy puntuales, sobre algunos de aquellos trabajos. El problema radica en que, al margen de algunas aportaciones realizadas por los miembros del Grupo de Estudios de Historia de la Cartografía, y esencialmente para el caso de Cataluña ${ }^{10}$, los historiadores y los geógrafos apenas han dedicado atención a este tipo de cartografía. Por otra parte, estos trabajos fueron encomendados a Casañal por parte de municipios y sindicatos de regantes a título individual, y no existe un archivo único que conserve esta documentación. Además, diversos municipios y sindicatos de riego tienen

9. La literatura sobre la historia de los ferrocarriles en la España del siglo XIX es muy abundante, pero los estudios sobre los planos que acompañaban a los proyectos son muy escasos, como se desprende del artículo de Muñoz Rubio y Vidal Olivares (2001). Entre las excepciones, y para el caso de Galicia, puede destacarse el trabajo ya citado de Nárdiz Ortiz (1992: 433-459).

10. Véase <http://www.ub.edu/gehc/index.php/es/>. 
constancia de la formación de los planos, pero muchos se han extraviado o, lamentablemente, destruido, al perder valor de uso. Por último, puede añadirse que, a la muerte de Casañal, desaparecieron el Centro-Geodésico Topográfico y su archivo. No obstante, se puede asegurar que, hasta la finalización del Bosquejo, había formado planos parcelarios de siete sindicatos de riego y municipios zaragozanos, como mínimo ${ }^{11}$.

Estas son las fuentes que Casañal cita, pero también realizó comprobaciones sobre el terreno — como había señalado en su propuesta- y se puso en contacto con diversos ayuntamientos para recabar algunas informaciones o comprobar otras. El 21 de octubre de 1898, informó a la DPZ acerca de que, para poder terminar los trabajos, tenía que recibir "las contestaciones al interrogatorio» que había enviado a algunos pueblos ${ }^{12}$.

\section{Los resultados}

\subsection{El Bosquejo}

En noviembre de 1899, Casañal presentó el Bosquejo a Leopoldo Anglés, presidente de la DPZ, pero de este documento no se tienen más noticias. Posiblemente se tratara de un primer esbozo bastante incompleto, que su autor debió mostrar para cumplir con el plazo a que se había comprometido, pues la primera versión impresa es de 1902 (figura 4).

Se trata de un documento al ferroprusiato, a escala 1:200.000, que la Diputación aprobó imprimir por unanimidad y que presenta notables diferencias con la versión definitiva del año siguiente (figura 5). En esta última, se incluyen algunas carreteras y vías férreas en proyecto, caminos de herradura, topónimos y orónimos, altitudes de poblaciones o la delimitación de los partidos judiciales, que no aparecían en la anterior. También se han corregido altitudes de poblaciones; se representan como carreteras en proyecto algunos caminos — probablemente a consecuencia de disposiciones oficiales—; se delimitan con mayor precisión los límites provinciales y del término de Petilla de Aragón, y se incluye el enclave de Bastanes de Petilla. Algunas de las modificaciones - como las referidas a límites - se deben a que Casañal elaboró la primera versión tomando como referencia, casi exclusivamente, informaciones del Atlas que no eran correctas. Así pues, la versión de 1902 era un documento provisional - aunque la DPZ aprobara su impresión-, que sería revisado minuciosamente para su publicación en $1903^{13}$.

La versión definitiva, de $140 \mathrm{~cm} \times 134 \mathrm{~cm}$, a la misma escala que la anterior y publicada a cuatro colores, contiene toda la información necesaria para servir de base a los objetivos de Casañal. Recoge todas las poblaciones, distinguiendo cabezas de partidos judiciales — delimitados estos por masas de color y de

11. La información sobre los mismos se localiza en el Archivo del Canal Imperial de Aragón.

12. ADPZ, Libro de actas, 1898, sign. 64.

13. ADPZ, Libro de actas, 1899, sign. 64, y 1902, sign. 68. 


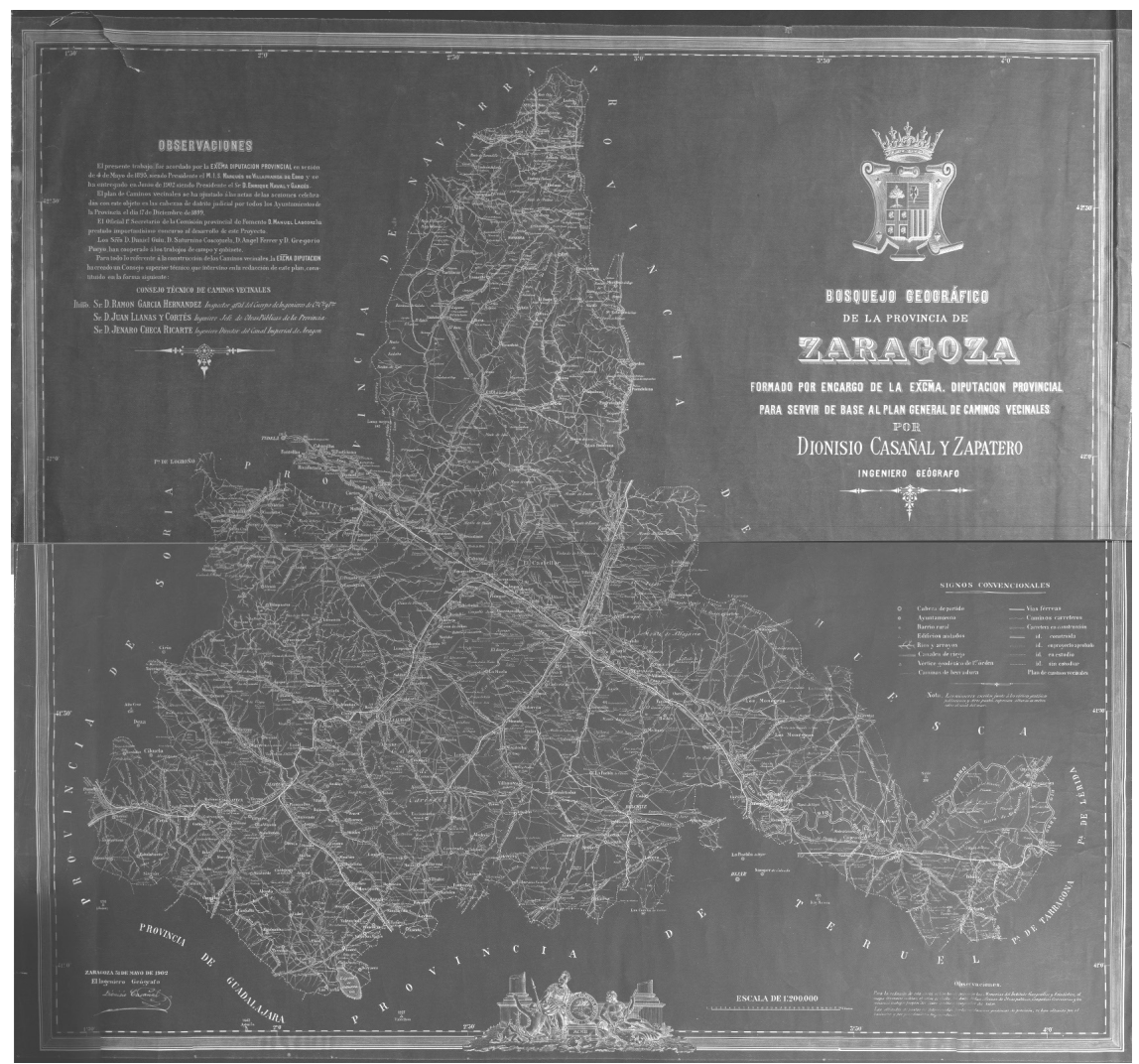

Figura 4. Bosquejo geográfico de la provincia de Zaragoza (1902), 1:200.000.

Fuente: Archivo de la Diputación Provincial de Zaragoza, Planoteca, sign. MP-75.

forma aproximada, como en el mapa de Coello-, ayuntamientos y barrios rurales, así como numerosos edificios aislados. Representa las vías de comunicación clasificándolas en carreteras (construidas, en construcción, en proyecto aprobado, en estudio y sin estudiar), caminos carreteros y de herradura, y vías férreas construidas y en proyecto. En la red hidrográfica, se diferencia la importancia de los cursos con caudal constante (ríos) o temporal (arroyos, barrancos, etc.) por anchura y se incluyen los canales de riego, las balsas y las lagunas. No existe representación del relieve de ningún tipo, pero se recogen numerosos orónimos, distinguiendo sierras, collados, cabezos, etc. También se indican las altitudes de numerosos lugares, aunque no de todas las poblaciones. Las altitudes no determinadas por nivelaciones geodésicas de precisión fueron calculadas por medio del barómetro y de procedimientos trigonométricos.

Si la representación de poblaciones, cursos de agua, carreteras, caminos y ferrocarriles construidos resulta muy detallada, el trazado de las líneas férreas 


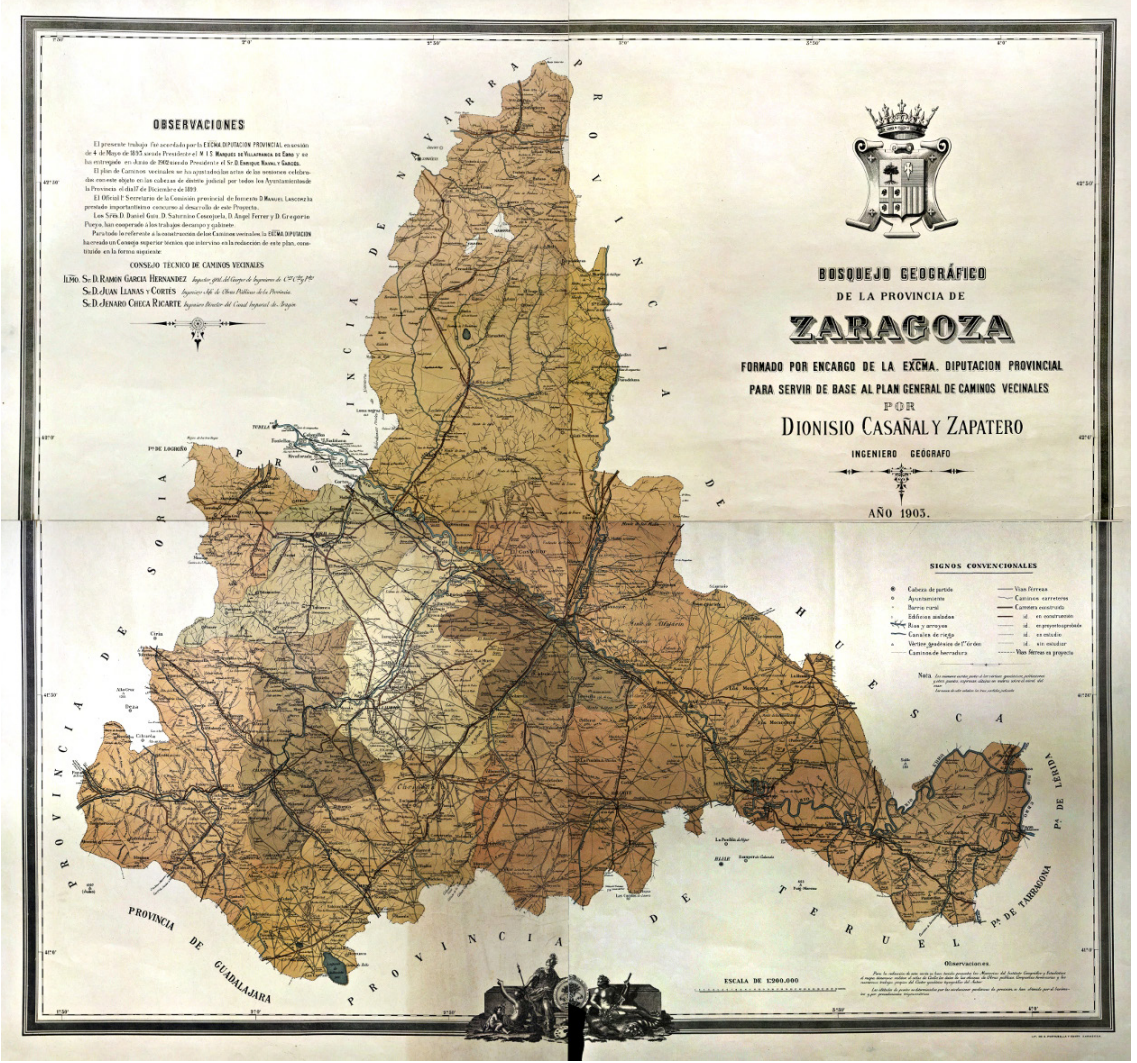

Figura 5. Bosquejo geográfico de la provincia de Zaragoza (1903), 1:200.000.

Fuente: Archivo de la Diputación Provincial de Zaragoza, Planoteca, sign. MP-94.

en construcción o en proyecto es más deficiente o de poca utilidad, pues la aprobación definitiva del trazado de algunas o su construcción se demorarían bastantes años. Por ejemplo, la iniciativa de la línea Gallur-Sádaba databa de 1887, pero las obras no comenzarían hasta 1912 (Lambán Montañés, 1984; Macías Muñoz, 2005). Algo similar sucede con el tramo Calatayud-Torrelapaja, de la línea Santander-Mediterráneo. A finales del siglo xix habían comenzado las obras en la provincia de Santander, pero el trazado definitivo no sería aprobado hasta 1912 y el tramo Calatayud-Soria por Torrelapaja se finalizaría en 1929. A consecuencia de estas vicisitudes, el trazado dibujado por Casañal es incompleto - no llega al límite de la provincia- y tampoco se corresponde con el definitivo ${ }^{14}$. Por otra parte, algunas líneas dibujadas (Cariñena-Daroca o Cariñena-Ricla) nunca se construirían, como ya se ha comentado.

14. Éste puede observarse en Dobeson (1988: 209-212). 
Junto al mapa y al Plan General de Caminos Vecinales, Casañal también se había comprometido a presentar otros documentos: una triangulación de la provincia apoyada en la Red Geodésica de Primer Orden establecida por el IGE y una memoria con los datos estadísticos sobre riqueza de la provincia que fueran precisos para razonar la urgencia y la necesidad de los caminos propuestos. Ni en las actas de las sesiones de la Diputación, ni en los fondos del ADPZ se ha localizado información alguna sobre ambas. En el Bosquejo solo se incluyen los vértices geodésicos de la Red de Primer Orden, cuya observación ya había ejecutado el IGE. Pero lo cierto es que ambos trabajos no eran necesarios para cumplir su objetivo. La triangulación se habría requerido para formar un mapa topográfico, no un «bosquejo». Y la memoria carecía de sentido al diseñarse el Plan General de Caminos Vecinales por otro procedimiento, como se verá a continuación. A pesar de la ausencia de estos trabajos, la DPZ quedó «altamente satisfecha y complacida por la meritoria labor realizada» y acordó por unanimidad nombrar a su autor Hijo Predilecto de la provincia. Tal vez influyera en esta decisión el hecho de que Casañal, como muestra de "cariño y afecto a la provincia», renunciara a cobrar una gran parte de las 8.000 pesetas presupuestadas. Por esta razón, la Diputación acordó entregarle 500 ejemplares de los 2.000 que había aprobado imprimir, para que hiciera «el uso que estimara conveniente» de ellos ${ }^{15}$.

\subsection{El Plan General de Caminos Vecinales de 1900 y su azaroso desarrollo}

Hasta aquí el análisis del Bosquejo, pero el hecho de que fuera realizado para servir de base a un plan general de caminos vecinales aconseja referirse, aunque sea brevemente, a los resultados del mismo. Como ya se ha señalado, en noviembre de 1899, Casañal presentó una primera versión del Bosquejo y añadió que le faltaba cumplir la segunda parte del compromiso contraído: "el estudio de un plan general de caminos vecinales». Con objeto de recabar la información necesaria para diseñarlo, la DPZ aprobó su idea de que los ayuntamientos presentaran propuestas en reuniones que se organizarían en las cabezas de los partidos judiciales. Con estos datos, la Diputación fijaría el plan definitivo que debería aprobar el gobernador civil conforme a la normativa vigente. Para asesorar a la Diputación en los aspectos técnicos, y por iniciativa de Casañal, se creó una comisión de especialistas formada por tres ingenieros: Ramón García Hernández, inspector jefe del Cuerpo de Ingenieros de Caminos, Canales y Puertos; Mariano Royo, director del Canal Imperial de Aragón - que sería reemplazado por Jenaro Checa, su sucesor al frente del Canal, al fallecer aquel mismo año-, y Juan Llanas, jefe de Obras Públicas de la provincia ${ }^{16}$.

En este punto, hay que hacer un inciso para señalar que el plan propuesto por Casañal se enmarcaba claramente en el ideario regeneracionista de la época

15. ADPZ, Libro de actas, 1902, sign. 68, y 1898, sign. 64.

16. ADPZ, Libro de actas, 1899, sign. 65; 1900, sign. 66, y Fondo Fomento, caja XIV-931. 
por dos razones. El enfoque coincidía con la idea de regenerar España, que consistía básicamente en "actuar con los instrumentos de la ingeniería para mejorar las condiciones materiales del territorio»: política hidráulica en beneficio de la agricultura, líneas de ferrocarril y red viaria de ámbito local (Navas y Ferrer, 2007: 141). En este sentido, y ajustando las posibilidades económicas a las necesidades de desarrollo y equilibrio territorial,

[...] los planes de caminos vecinales apostaban por una red compuesta por vías de corto recorrido afluentes de las carreteras estatales, de algunas secundarias, y también de ciertas estaciones de ferrocarril que debían llegar hasta los núcleos rurales dispersos. El propósito era buscar la distancia más corta entre el núcleo de población y la red viaria existente, a través de unas líneas que dotaran de accesos individualizados al sistema de asentamientos urbanos diseminados por el territorio. (Navas y Ferrer, 2013: 35)

Por otra parte, la elección de Mariano Royo y Ramón García Hernández como miembros de la comisión técnica confirmaba aquel enfoque. Ambos habían realizado obras y actuaciones muy notables en el ámbito hidráulico, siendo pioneros en las demandas de implantación de una política hidráulica en las que se inspiraría Joaquín Costa (Sáenz Ridruejo, 1990; Germán Zubero, 1999b; Jaime Lorén, 2009). Aunque también habían manifestado su preocupación sobre la importancia de los caminos vecinales para impulsar el desarrollo agrícola. Royo había reclamado una especial protección de la agricultura por parte del Estado. Esta protección no debía ser solo para el cultivo, sino que también tenía que estar orientada «a facilitar la extracción de los productos hasta sus mercados naturales» (Royo, 1899: 137). A su vez, García Hernández había redactado un plan de rehabilitación de los caminos vecinales existentes para la Diputación Provincial de Teruel en 1876 y había publicado «Algunas consideraciones sobre la manera de completar con economía el Plan General de Caminos» en la Revista de Obras Públicas, donde se mostraba partidario de fomentar los que llamaba «caminos económicos [...] dada la situación del Tesoro Público y el de las Provincias y Municipios» (García, 1881: 62), y, siendo miembro de la Comisión Técnica, publicó otro artículo en el que insistía en la misma idea para que el mayor número posible de pueblos pudiera acceder con facilidad y lo más pronto posible a conectarse con la red de carreteras y ferrocarriles (García, 1903) ${ }^{17}$.

En marzo de 1900, tras recibir las proposiciones de los ayuntamientos, la DPZ aprobó por unanimidad las bases para la construcción de los caminos - para cuya redacción Casañal había tenido «muy en cuenta» las elaboradas por la Diputación de Barcelona en 1888 y 1890 - y una relación provisional de 105 caminos, con una longitud total de $1.455 \mathrm{~km}^{18}$. Dicha relación fue elaborada por Casañal y por la Comisión Técnica tras examinar las propuestas y

17. En ambos artículos también plasmó sus concepciones acerca de la ingeniería viaria y propuso soluciones encaminadas a buscar la economía y el ahorro de medios y materiales.

18. Las bases y la relación de los caminos se localizan en ADPZ, Fondo Fomento, caja XIV-931. 
teniendo presentes el estado «de las carreteras generales y provinciales construidas, en construcción y pendientes de estudio». La Diputación llegó a afirmar que se había logrado «no dejar aislado del concierto general ningún pueblo de la provincia». Respecto a la financiación de las obras, la DPZ subvencionaría los gastos de habilitación con el $50 \%$ del presupuesto y los ayuntamientos se comprometerían a "concurrir con la prestación personal» a la ejecución de las obras y a sufragar los gastos de las expropiaciones si las hubiere ${ }^{19}$.

La DPZ aprobó el Plan en 1900, pero durante más de tres años estuvo prácticamente paralizado, debido, fundamentalmente, a las dificultades económicas que padecía la institución ${ }^{20}$. La reactivación se produjo gracias a la Real Orden de 13-8-1903, que planteó la necesidad de que cada provincia propusiese un plan de caminos «relativamente modesto», de $200 \mathrm{~km}$, en el que se incluyeran aquellos que enlazaran «los pueblos aislados a las vías férreas y a las carreteras generales». El mes siguiente, la Real Orden de 5-9-1903 dispuso que su construcción comenzaría en diez provincias seleccionadas previo concurso y en las que ofrecieran auxilios superiores al $50 \%$ del coste de las obras ${ }^{21}$. La Comisión Provincial decidió que la DPZ y los municipios aportaran el 55\%, para asegurar la selección, y que los $200 \mathrm{~km}$ se adjudicaran entre los pueblos que ofrecieran mayores auxilios. El 28 de septiembre, la Diputación aprobó una relación de 32 caminos, entre los que se encontraban algunos no contemplados en 1900, debido a las nuevas condiciones dictadas y a que algunos ayuntamientos se comprometieron a aportar mayores auxilios ${ }^{22}$.

Las obras se inauguraron en octubre, pero la precipitada tramitación del proceso provocaría que surgiesen numerosos problemas - proyectos y presupuestos que no se habían podido ultimar por falta de tiempo, sobrecostes, imposibilidad de algunos ayuntamientos para aportar los auxilios ofrecidos, desacuerdos entre municipios, discrepancias entre la DPZ y la Dirección Gene-

19. ADPZ, Libro de actas, 1900, sign. 66.

20. ADPZ, Libro de actas, 1901, sign. 67; 1902, sign. 68; 1903, sign, 69, y Fondo Fomento, caja XIV-931. Sobre la penuria financiera de las corporaciones locales en general y de las diputaciones en particular, penuria que les impedía cumplir de modo eficaz las competencias que tenían encomendadas, ver Santana Molina (1989: 256-262).

21. Ambas reales órdenes habían sido dictadas por Rafael Gasset, ministro de Agricultura, Industria, Comercio y Obras Públicas. Gasset propugnó la reconstrucción económica del país tras el «desastre de 1898» mediante el impulso de las obras públicas, y especialmente de las hidráulicas con destino al riego. Si bien consideraba que estas últimas constituían uno de los medios principales para contribuir al desarrollo de la producción agrícola, también era consciente de que las actuaciones en este ámbito eran inseparables de la facilidad y de la economía de transporte. En 1911, presentaría varios proyectos de ley de obras públicas, entre ellos el de construcción de caminos vecinales. La debilidad de la hacienda pública y las tensiones entre los partidos parlamentarios, que dificultarían la aprobación de presupuestos extraordinarios para acometer dichas obras, provocarían que los resultados quedaran muy alejados de sus ambiciosos objetivos (Villanueva Larraya, 1987 y 1990), como sucedió con sus primeras disposiciones.

22. De los 32 seleccionados, solamente 13 estaban incluidos en el Plan General de Caminos Vecinales de 1900. Ver ADPZ, Fondo Fomento, caja XIV-931, y ADPZ, Libro de actas, 1903, sign. 69. 
ral de Obras Públicas acerca de los términos y las condiciones en que la primera debía abonar las cantidades comprometidas, etc.- que paralizaron algunas obras e impidieron el inicio de otras ${ }^{23}$.

En esta situación, la aprobación de la Ley de Caminos Vecinales de 30-71904 obligó a revisar nuevamente el Plan. Por primera vez aparecía, de forma explícita, el criterio de que los caminos vecinales no solo eran responsabilidad de los ayuntamientos, sino que el Estado y las diputaciones también tenían que intervenir decididamente en su construcción (Navas y Ferrer, 2007). En las disposiciones transitorias se disponía la continuación de las obras en los caminos ya iniciados y, "previa la revisión del plan» por el Ministerio tras escuchar a las diputaciones y a los ingenieros jefes de Obras Públicas, en el resto de los que componían las relaciones de los $200 \mathrm{~km}$.

En octubre, la DPZ solicitó la continuación de las obras en los tres caminos en los que ya habían comenzado y el inicio en otros 16 de los que ya se habían firmado los contratos con los ayuntamientos. Para completar la relación abrió un nuevo concurso para que los ayuntamientos concretasen las propuestas de auxilio. Solamente recibió siete solicitudes y en dos casos las corporaciones municipales no se responsabilizaban de cumplir todas las exigencias. La Diputación propuso al Ministerio la relación de los caminos aprobados en octubre y de los nuevos cinco que cumplían los requisitos. La Real Orden de 27-3-1905 aprobó el nuevo plan que comprendía solamente $102 \mathrm{~km}$. Al año siguiente, el Real Decreto de 15-4-1906 intentó reimpulsar los trabajos y la DPZ aprobó una nueva lista con otros 16 caminos $^{24}$. Pero el ritmo de los trabajos continuó siendo extremadamente lento. En 1910, solo se habían finalizado $34 \mathrm{~km}$ de caminos vecinales (Germán Zubero, 1999a: 346) y, en 1917, todavía existían 104 pueblos incomunicados en la provincia — aquellos que no tenían comunicación por carretera, camino transitable o ferrocarril, y que se hallaban a más de 3,5 km de una vía cualquiera—, entre ellos, 16 de más de 1.000 habitantes (Biescas Ferrer, 1985: 108).

\section{Conclusiones}

A finales del siglo xIx, la escasez de caminos vecinales en la provincia de Zaragoza dificultaba enormemente la comercialización de la producción agraria de los núcleos rurales, y la crisis agraria finisecular agravaba la situación. En este contexto, y ante la ausencia de cartografía de base - no existía ni un mapa topográfico ni un mapa corográfico provincial actualizado-, Dionisio Casañal propuso a la DPZ formar una carta geográfica que sirviera de base cartográfica para la preparación de un plan general de caminos vecinales en la provincia con objeto de minimizar el problema. El resultado fue el Bosquejo geográfico de la provincia de Zaragoza. La carta, a escala 1:200.000, fue esencialmente un trabajo de gabinete y las operaciones de campo escasas, ya que Casañal lo realizó

23. ADPZ, Fondo Fomento, caja XIV-931.

24. ADPZ, Fondo Fomento, caja XIV-931, y Libro de actas, 1904, sign. 70, y 1905, sign. 71. 
prácticamente gratis — su autor, diputado provincial que defendía los planteamientos regeneracionistas, era consciente de las dificultades económicas de la Diputación-, aprovechando la infraestructura y el personal de su empresa, el Centro Geodésico-Topográfico, y a partir de las fuentes cartográficas existentes que podían proporcionarle mayor información para lograr su objetivo.

El resultado final no fue un mapa muy preciso, sino un «bosquejo» - como Casañal lo intituló-, en el que se detallaban correctamente la localización de las poblaciones y el trazado de las carreteras o de los caminos vecinales existentes o en proyecto, así como la red hidrográfica. Sin embargo, no contó con representación del relieve - solamente incluía orónimos, diferenciando sierras, collados, etc._-, ni especificó la altitud de todas las poblaciones. Los trazados de las líneas ferroviarias en construcción o en proyecto tampoco se correspondieron, evidentemente, con los que fueron modificados posteriormente o no llegaron a construirse.

A pesar de todo, el Bosquejo fue recibido con satisfacción por la Diputación. Por una parte, disponía de una carta geográfica provincial actualizada. Tendrían que transcurrir muchos años para contar con un mapa comparable. En 1936, y finalizado el levantamiento del Mapa topográfico nacional del territorio de la provincia, el Instituto Geográfico publicó el mapa Provincia de Zaragoza, a escala 1:200.000, que incluía las vías de comunicación (carreteras clasificadas en tres órdenes, caminos vecinales y ordinarios), ferrocarriles (vía ancha y estrecha) y red hidrográfica (ríos, arroyos, canales, acequias, lagunas, etc.). Como grandes novedades, incorporaba la delimitación de los términos municipales y el relieve, representado mediante curvas de nivel equidistantes $100 \mathrm{~m}$. Por otra parte, el Bosquejo resultaría una herramienta fundamental para diseñar el Plan General de Caminos Vecinales de 1900. Con la información que contenía, la DPZ analizó la situación existente y elaboró la lista de los caminos a construir.

En lo que respecta al Plan, este encontró numerosas dificultades para su ejecución, a causa, fundamentalmente, de problemas económicos y políticos. La penuria financiera de la Diputación y los municipios impidió ponerlo en marcha hasta 1903, cuando el ministro Gasset decidió subvencionar buena parte del coste de las obras, cuyos estudios técnicos y cuya ejecución correrían a cargo de la Delegación Provincial de Obras Públicas. No obstante, a partir de aquel momento, los problemas persistirían. Las corporaciones locales continuaron sin disponer de suficientes recursos para hacer frente a sus compromisos. El Ministerio de Fomento encontró enormes dificultades para vencer la resistencia parlamentaria para aprobar los presupuestos extraordinarios necesarios, en un contexto económico difícil, tras el "desastre» de 1898, y una situación política muy inestable ${ }^{25}$. Por ello, los resultados del Plan General de Caminos Veci-

25. A modo de ejemplo, puede comentarse que, en los menos de tres años que mediaron entre la Real Orden de 13-8-1903 - la primera iniciativa del ministro Gasset para impulsar planes de caminos vecinales - y el Real Decreto de 15-4-1906 — que intentó infructuosamente reimpulsar los trabajos_, existieron siete gobiernos en España (Urquijo Goitia, 2008: 88-92). 
nales, que sufrió diversas modificaciones, fueron decepcionantes —en 1910, solo se habían finalizado $34 \mathrm{~km}$ de caminos- y, consecuentemente, la aportación real del Bosquejo al proceso de modernización de la red viaria zaragozana fue mínima.

\section{Referencias bibliográficas}

Alzola y Minondo, Pedro de (1899). Historia de las obras públicas en España. Madrid: Colegio de Ingenieros de Caminos, Canales y Puertos, 2001.

BiesCAs FerRer, José Antonio (1985). El proceso de industrialización en la región aragonesa en el período 1900-1920. Zaragoza: Institución Fernando el Católico.

Dobeson, Kenneth M. (1988). El Ferrocarril Santander-Mediterráneo. Madrid: Aldaba.

Font i Garolera, Jaume (1999). La formación de les xarxes de transport a Catalunya (1761-1935). Vilassar de Mar: Oikos-Tau.

FonTANA, Josep (1986). «La dinámica del mercado interior: (Algunas reflexiones a propósito del crecimiento de Santander)». En: Martínez VAra, Tomás (ed.). Mercado y desarrollo económico en la España contemporánea. Madrid: Siglo XXI, 85-96.

GarCíA, Ramón (1881). «Algunas consideraciones sobre la manera de completar con economía el plan general de carreteras». Revista de Obras Públicas, I (6, 7 y 8), 61-64, 73-76 y 85-85.

- (1903). «Caminos vecinales». Revista de Obras Públicas, II (1461), 519-521.

García-Baquero, Manuel (1981). Historial del Mapa Militar Itinerario de España. Madrid: Servicio Geográfico de Ejército.

Germán Zubero, Luis (1999a). «El transporte por carretera en Aragón durante la etapa del ferrocarril». En: Magallón, M. a Ángeles (coord.). Caminos y comunicaciones en Aragón. Zaragoza: Institución Fernando el Católico, 341-349.

- (1999b). Obras públicas e ingenieros en Aragón durante el primer tercio del siglo XX. Zaragoza: Institución Fernando el Católico / Colegio de Ingenieros de Caminos, Canales y Puertos (Aragón).

- (1999c). «Red ferroviaria y evolución del comercio interregional de la economía aragonesa, 1875-1930». En: Muñoz Rubio, Miguel; Sanz Fernández, Jesús y Vidal Olivares, Javier (eds.). Siglo y medio del ferrocarril en España, 1848-1998: Economía, industria y sociedad. Madrid: Fundación de los Ferrocarriles Españoles, 517-532.

- (2012). Historia económica del Aragón contemporáneo. Zaragoza: Prensas Universitarias de Zaragoza.

Germán, Luis y ForCadell, Carlos (1988). «La crisis finisecular de la agricultura interior: El caso de Aragón». En: Garrabou, Ramon (ed.). La crisis agraria de fines del siglo XIX. Barcelona: Crítica, 69-93.

Gómez Mendoza, Antonio (1991). «Las obras públicas (1850-1935)». En: Comín, Francisco y Martín AceÑa, Pablo (dirs.). Historia de la empresa pública en España. Madrid: Espasa Calpe, 177-204.

Gómez Pérez, José (1971). «El Atlas de España y sus talleres de grabado (Empresa Madoz-Coello)». Anales del Instituto de Estudios Madrileños, VII, 401-419.

Herranz Loncan, Alfonso (2004). La dotación de infraestructuras en España (18441935). Madrid: Banco de España.

- (2008). Infraestructuras y crecimiento económico en España (1850-1935). Madrid: Fundación de los Ferrocarriles Españoles. 
JAIMe LoRÉn, José M. a de (2009). «El ingeniero Ramón García Hernández y las obras públicas en España a fines del s. XIX». Llull: Revista de la Sociedad Española de Historia de las Ciencias y de las Técnicas, 32, 73-101.

Lambán Montañés, Javier (1984). «El ferrocarril Sádaba-Gallur». Cuadernos de Aragón, 18, 77-111.

Macías Muñoz, Olga (2005). «Los ferrocarriles de vía estrecha en Navarra y el Valle del Ebro». En: Muñoz Rubio, Miguel (dir.). Historia de los Ferrocarriles de Vía Estrecha en España. Vol. I, Empresas y Marco Regional. Madrid: Fundación de los Ferrocarriles Españoles, 241-308.

Magallanes Pernas, Luis Antonio (2014). "Cartografía itineraria del Cuerpo de Estado Mayor del Ejército de España». Revista Ejército, 884, 108-115.

Menéndez de Luarca y Navía Osorio, José Ramón (2000). La construcción del territorio: Mapa histórico del noroeste de la Península Ibérica. Barcelona: Lunwerg.

Moreno SARdÀ, Amparo (dir.) (1998). Xarxes de comunicación a Catalunya. Passat, present i futur [CD-ROM]. Bellaterra: Universitat Autònoma de Barcelona.

Muñoz Rubio, Miguel y Vidal Olivares, Francisco Javier (2001). «Los ferrocarriles en la historiografía española». Transportes, Servicios y Telecomunicaciones, 1, 81-111.

Nárdiz Ortiz, Carlos (1992). El territorio y los caminos en Galicia: Planos históricos de la red viaria. Madrid: Colegio de Ingenieros, Canales y Puertos / Xunta de Galicia.

Navas [y Ferrer], Teresa (2007). Història de les carreteres del territori de Barcelona. Barcelona: Diputació de Barcelona.

- (2012). Planificació, construcció i mobilitat: La modernització de la xarxa viària de la regió de Barcelona. 1761-1969. Barcelona: Departament de Geografia Física i Anàlisi Geogràfica Regional. Universitat de Barcelona. Tesis doctoral.

- (2013). «Infraestructuras viarias desde la perspectiva histórica: El caso de la formación de la red de carreteras en la región de Barcelona (1761-1969)». Revista Transporte y Territorio, 8, 27-44.

Pinilla Navarro, Vicente (1995). Entre la inercia y el cambio: El sector agrario aragonés, 1850-1935. Madrid: Ministerio de Agricultura, Pesca y Alimentación.

Quirós Linares, Francisco (2010). «La cartografía de la metrópoli en el Atlas de España y sus posesiones de Ultramar (1847-1870), de Francisco Coello: Características, fuentes y colaboradores". Ería, 81, 63-92.

Rodríguez Lázaro, Francisco J.; Coronado Tordesillas, José M.a; Ruiz Fernández, Rita y Garcilaso de la Vega MuÑoz, Juan (2007). Análisis y valoración del patrimonio histórico de las carreteras españolas, 1748-1936. Madrid: Ministerio de Fomento.

Royo, Mariano (1899). «Las obras públicas y la riqueza agrícola». Revista de Obras Públicas, I (1229), 137-139.

Sáenz Ridruejo, Fernando (1990). Ingenieros de Caminos del siglo XIX. Madrid: Colegio de Ingenieros de Caminos, Canales y Puertos.

Santana Molina, Manuel (1989). La Diputación Provincial en la España decimonónica. Madrid: Instituto Nacional de Administración Pública.

Soria y Puig, Arturo (1993). El camino a Santiago: Vias, estaciones y señales. Madrid: Ministerio de Obras Públicas y Transporte.

Soria y Puig, Arturo y Menéndez de Luarca, José Ramón (1994). «El territorio como artificio cultural: Corografía histórica del Norte de la Península Ibérica». Ciudad y Territorio: Estudios Territoriales, 99, 63-94. 
Tedde de Lorca, Pedro (1978). «Las compañías ferroviarias en España, 1855-1935». En: Artola, Miguel (dir.). Los ferrocarriles en España, 1844-1943. Vol. II, Los ferrocarriles y la economía. Madrid: Banco de España, 9-354.

Uriol Salcedo, José I. (1992). Historia de los caminos de España. Vol. II, Siglos XIXy $X X$. Madrid: Colegio de Ingenieros de Caminos, Canales y Puertos.

URquijo Goitia, José Ramón (2008). Gobiernos y ministros españoles en la edad contemporánea. Madrid: CSIC.

URTEAga, Luis y NADAL, Francesc (2001). Las series del mapa topográfico de España a escala 1:50.000. Madrid: Instituto Geográfico Nacional.

- (2007). «Las políticas de intervención sobre el territorio y el papel de la cartografía: El caso del Mapa Topográfico de España (1856-1923)». En: Lafuente, Antonio; Cardoso, Ana M. ${ }^{a}$ y Saraiva, Tiago (eds.). Maquinismo ibérico. Madrid: Doce Calles, 401-418.

Villanova, José Luis (2011a). «Dionisio Casañal y Zapatero: Del catastro a la topografía (1864-1878)». En: Montaner, Carme; Nadal, Francesc y Urteaga, Luis (eds.). Cartografia i agrimensura a Catalunya $i$ Balears (segles XIX-XX). Barcelona: Institut Cartogràfic de Catalunya, 209-223.

- (2011b). «El Plano de Córdoba (1884) de Dionisio Casañal y Zapatero». Cuadernos Geográficos de la Universidad de Granada, 49, 123-152.

- (2014). «La cartografía de las plazas fuertes españolas: El caso del plano de Pamplona de 1882». Treballs de la Societat Catalana de Geografia, 77, 345-368.

Villanova, José Luis; Palanques, María Luisa y Calvo, Miguel (2015). «El plano de Vitoria de Dionisio Casañal (1888)». Scripta Nova. Revista Electrónica de Geografía y Ciencias Sociales [en línea], XIX (501). <http://www.ub.es/geocrit/sn/sn-501. pdf>.

Villanueva Larraya, Gregoria (1987). «Rafael Gasset: "La política hidráulica” en la acción de gobierno". Revista de la Facultad de Geografía e Historia, 1, 439-459.

- (1990). «Apuntes para una biografía política de Rafael Gasset, un liberal regeneracionista». Espacio, Tiempo y Forma, Serie V, Ha Contemporánea, 3, 159-171.

WaIs, Francisco (1956). "Origen y desarrollo de los ferrocarriles aragoneses». Zaragoza, III, 85-112. 